\title{
Intravitreal anti-VEGF treatment for choroidal neovascularization secondary to traumatic choroidal rupture
}

\author{
T. Barth ${ }^{1 *}$ D, F. Zeman², H. Helbig ${ }^{1}$ and M.-A. Gamulescu
}

\begin{abstract}
Background: So far only single cases with short follow-up have been reported on the use of intravitreal anti-VEGF for traumatic choroidal neovascularizations (CNV). This paper reports a large case series of patients with CNV secondary to choroidal rupture after ocular trauma receiving intravitreal anti-VEGF (vascular endothelial growth factor) injections.

Methods: Fifty-four patients with unilateral choroidal rupture after ocular trauma diagnosed between 2000 and 2016 were retrospectively evaluated. Eleven patients with CNV secondary to choroidal rupture were identified. Five eyes with traumatic secondary CNV were treated with anti-VEGF and were systematically analysed. The other 4 patients with inactive CNV underwent watchful observation.

Results: Four men and one woman with a mean age of 29 years (SD 12.4; range 19-45) had intravitreal anti-VEGF therapy for traumatic CNV. Another 4 patients with a mean age of 37 years (SD 6.6; range 31-46) presented with inactive CNV and did not receive specific treatment. In all 9 cases the mean interval between the ocular trauma and the diagnosis of CNV was 5.7 months (SD 4.75; range 2-12). In the treatment group per eye 4.2 injections (SD 3.2; range 1-8) were given on average. Four eyes were treated with bevacizumab and one eye with ranibizumab. Regression of CNV was noted in all eyes. In 4 eyes visual acuity (VA) improved, one eye kept stable visual acuity.

Conclusions: Here, we present the up to now largest case series of traumatic CNV membranes treated with antiVEGF injections with a mean follow-up period of 5 years. Intravitreal anti-VEGF therapy seems to be safe and effective for secondary CNV after choroidal rupture. Compared to exudative age-related macular degeneration fewer injections are needed to control the disease.
\end{abstract}

Trial registration: Retrospective registration with local ethics committee on 21 March 2019. Trial registration number is 19-1368-104.

Keywords: Secondary choroidal neovascularization, Choroidal rupture, Intravitreal anti-VEGF (vascular endothelial growth factor) treatment

\section{Introduction}

Traumatic choroidal neovascularization (CNV) occurs in patients with choroidal rupture following severe ocular injuries [1]. Compared to $\mathrm{CNV}$ in age-related macular degeneration (AMD) the course of traumatic neovascular membranes is relatively benign [1]. As choroidal neovascularizations are a consistent part of the ocular repair

\footnotetext{
*Correspondence: teresa.barth@ukr.de

'Department of Ophthalmology, University Medical Centre Regensburg,

Franz-Josef-Strauß-Allee, 1193053 Regensburg, Germany

Full list of author information is available at the end of the article
}

mechanism they usually regress spontaneously as part of the healing process $[2,3]$. The formation of persistent $\mathrm{CNV}$ with substantial loss of central vision happens in $5-25 \%$ of eyes with choroidal ruptures $[1,3]$. In the early phase (within 6 months of the injury) the CNV development is explained by an exuberant repair reaction or just persistence of the normal reparative neovascular tissue [1]. Late CNV formation (after one year of the injury) could either be caused by awakening of an old dormant $\mathrm{CNV}$ or more likely by a new $\mathrm{CNV}$ in an area of local weakness [1]. In earlier days selected case of traumatic

(c) The Author(s). 2019 Open Access This article is distributed under the terms of the Creative Commons Attribution 4.0 International License (http://creativecommons.org/licenses/by/4.0/), which permits unrestricted use, distribution, and 
CNV were treated by photodynamic therapy (PDT) $[4,5]$, laser photocoagulation [6] or submacular surgery [7]. After the introduction of intravitreal anti-VEGF (vascular endothelial growth factor) as therapy for exudative AMD some reports of antiangiogenic injections for $\mathrm{CNV}$ of other causes than AMD [8-10] and in younger populations [11] have been published. So far no larger only single case reports have been published on intravitreal injections for traumatic CNV [12-14]. Thus, it is important to collect data about the use of intravitreal anti-VEGF agents in individuals with this condition.

\section{Methods}

We identified 54 cases of choroidal rupture after ocular trauma, which were diagnosed between 2000 and 2016 at a large tertiary university eye hospital. Eleven patients with CNV secondary to choroidal rupture were identified and further evaluated. We divided the study population in two different groups. The first group $(n=5)$ included patients with active CNV lesion at initial presentation, that received intravitreal anti-VEGF injections. The second group $(n=4)$ consisted of eyes with inactive $\mathrm{CNV}$ at first presentation which did not receive specific treatment. The two remaining patients were excluded because of different treatment strategies (vitrectomy, PDT). The patients' medical records were retrospectively analysed. The following parameters were assessed: age, sex, type of injury, interval between trauma and CNV formation, treatment modalities and rate of recurrence. For anonymization the patients were grouped into different age groups (18-25 years, 26 -30 years, 31 - 40 years, 41 - 50 years.) The baseline examination included measurement of best corrected visual acuity (BCVA), slit lamp examination, ophthalmoscopy and retinal imaging. Diagnosis of CNV secondary to choroidal rupture was made clinically by at least two retina specialists and was confirmed by multimodal imaging of the posterior segment. ) Fundus fluorescein angiography (FFA) was achieved with Spectralis ${ }^{\circ}$ (Heidelberg Engineering, Heidelberg, Germany). On the basis of the FFA images the greatest linear dimension (GLD) of the lesion was calculated and the type, activity and location of the CNV was analysed (subfoveal: within the foveal avascular zone (FAZ), juxtafoveal: distance from FAZ $\leq 200$ $\mu \mathrm{m}$, extrafoveal: distance from FAZ $>200 \mu \mathrm{m})$. The decision of the treatment modality was made by at least two independent ophthalmologists and was based on the activity of the CNV lesion verified by leakage on FFA images. For the patients that underwent no specific CNV treatment no further optical coherence tomography (OCT) scans were performed. For the patients that underwent anti-VEGF treatment retinal imaging included also optical coherence tomography (OCT) at baseline and on all follow-up visits with a central 6-line radial OCT. The central foveal thickness (CFT) was calculated and the existence of intra- or subretinal fluid was noted. The definition of recurrence was reappearing activity either seen by leakage on FFA images or fluid on OCT scans at least three months after the last injection. The patients in the treatment group received at least one antiVEGF injection of either bevacizumab $(1.25 \mathrm{mg} / 0.05 \mathrm{ml})$ or ranibizumab $(0.5 \mathrm{mg} / 0.05 \mathrm{ml})$. There was no standardised treatment protocol. In every case the count, frequency and substance of anti-VEGF injections was chosen individually. The follow-up examination included Snellen's charts visual acuity, slit-lamp examination, funduscopy and retinal imaging. A conversion of the visual acuity equivalents attained using Snellen's charts to $\log$ MAR values was performed for statistical evaluation. The statistical investigation was achieved by using SPSS 23.0 (IBM, Chicago, USA). Categorical variables are listed as frequency counts and percentages. Continuous values are displayed as mean \pm standard deviation and range (min-max); Mean values were compared using Student's t-test. A p-value $<0.05$ was considered statistically significant.

\section{Results \\ Demographic and clinical data}

Overall, 54 patients were diagnosed with choroidal rupture after ocular trauma between 2000 and 2016. Eleven (20\%) of these patients developed secondary CNV. One patient in 2001 was treated with focal laser photocoagulation and PDT before the introduction of intravitreal anti-VEGF. Another patient underwent pars-plana-vitrectomy for CNVrelated vitreous haemorrhage. Of the 9 remaining cases, 5 eyes diagnosed between 2009 and 2016 with secondary traumatic $\mathrm{CNV}$ received intravitreal antiangiogenic treatment. In the 4 other eyes no specific medical treatment was recommended because of inactivity of the lesion at initial presentation. Overall $6(67 \%)$ right eyes and $3(33 \%)$ left eye were assessed. The study population involved $6(67 \%)$ men and 3 women (33\%). The mean age was 32 years (SD 10.3; range 19-46). Eight patients had a history of blunt trauma with one case of multiple facial fractures. The ninth patient presented with choroidal rupture after a penetrating eye injury with an intraocular foreign body. In every case the $\mathrm{CNV}$ development occurred within the early phase of one year after injury. The mean interval between the ocular trauma and the diagnosis of CNV was 5.7 months (SD 4.75; range 2-12). At baseline three patients reported decreased central vision with an average duration of symptoms of 10 weeks (SD 12; range 2-24). Six patients were diagnosed with CNV during posttraumatic routine follow-up examination. Table 1 summarizes the demographic and clinical data of the treatment group and the group without therapy.

\section{Morphologic and anatomical results}

At the initial diagnosis all 9 eyes presented with a classic $\mathrm{CNV}$ lesion. In 4 eyes the CNV was located extrafoveally (44\%), three CNV membranes were in the subfoveal 
Table 1 Clinical data of patients with traumatic choroidal neovascularization (CNV) treated with anti-VEGF [Group 1] and patient without CNV treatment [Group 2]

\begin{tabular}{llll}
\hline Patients with traumatic CNV & Group 1 (intravitreal anti-VEGF) & Group 2 (no treatment) & Both groups \\
\hline No. of cases & 5 & 4 & 9 \\
Right eyes & $4(80 \%)$ & $2(50 \%)$ & $6(67 \%)$ \\
Age (years) & $29 \pm 12.4$ & $37 \pm 6.6$ & $32 \pm 10.3$ \\
Sex (men) & $4(80 \%)$ & $2(50 \%)$ & $6(67 \%)$ \\
Time between trauma and CNV (months) & $6.0 \pm 4.6$ & $5.3 \pm 5.9$ & $5.7 \pm 4.75$ \\
GLD of the CNV ( $\mu$ m) & $1345 \pm 865$ & $1215 \pm 535$ & $1296 \pm 717$ \\
Initial logMAR BCVA & $0.80 \pm 0.32$ & $1.2 \pm 0.35$ & $0.98 \pm 0.38$ \\
Final logMAR BCVA & $0.42 \pm 0.26$ & $1.1 \pm 0.35$ & $0.66 \pm 0.43$ \\
Follow-up (months) & $62 \pm 32$ & $82 \pm 66$ & $69 \pm 44$ \\
\hline
\end{tabular}

$B C V A$ Best corrected visual acuity, GLD Greatest linear diameter, CFT Central foveal thickness

Data present mean \pm SD or absolute number (percentage)

region (33\%) and 2 patients showed juxtafoveal location (22\%). Four eyes (44\%) with an inactive and scarred $\mathrm{CNV}$ lesion at initial presentation underwent watchful observation and did not receive specific medical treatment. Five eyes (56\%) showed manifest CNV activity detected by apparent leakage on FFA images and were treated with intravitreal anti-VEGF injections. In these eyes with CNV the baseline OCT scan showed a mean CFT of $645 \mu \mathrm{m}$ (SD 359, range 329-1240). After completion of anti-VEGF-therapy an average CFT of $403 \mu \mathrm{m}$ (SD 145, range 214-586) was measured at the last visit. This reduction of CFT after anti-VEGF treatment was not statistically significant $(p=0.089)$. FFA was performed in all 9 eyes and showed a mean GLD of the neovascular membrane of $1296 \mu \mathrm{m}$ (SD $717 \mu \mathrm{m}$, range 451-2725). Details about the CNV morphology in the treatment group as well are listed in Table 2. Figure 1 shows fundus and FFA images of a 19-year-old male with traumatic CNV in the left eye. The OCT scans of a 23-year-old man with traumatic CNV in the right eye before treatment and 4 weeks after the last injection are presented in Fig. 2.

\section{Treatment characteristics and recurrence rates}

The 5 patients with an active CNV lesion received intravitreal antiangiogenic treatment as primary treatment for CNV. On average, 4.2 injections (SD 3.1, range 1-8) were given per eye. Four eyes were treated with bevacizumab, 1 eye with ranibizumab. The decision about the injection scheme was made individually and was dependent on the size and activity of the lesion. In two (40\%) eyes, which received a single anti-VEGF load at the beginning, this single injection was sufficient to control the disease. The other 3 $(60 \%)$ eyes were treated with an upload of three monthly injections. After a three month treatment pause 2 of these patients needed another 2 or 3 injections to control the $\mathrm{CNV}$ activity over a period of 3 to 6 months. In one of these patients recurrence occurred after a six-month treatment pause and was controlled by a single re-injection. The third patients with an initial upload of three injections had a two month treatment pause and another five injections afterwards over a period of half a year.

\section{Functional outcome in the treatment group}

Before the anti-VEGF treatment the mean $\operatorname{logMAR}$ BCVA in the treatment group was 0.80 (SD 0.32, range 0.4-1.3), which equates to a decimal visual acuity (VA) of 0.16. The logMAR BCVA in the cases without further therapy was 1.2 (SD 0.35, range 0.7-1.5) equivalent to a decimal VA of 0.06 . As expected the group of patients with an inactive, scared CNV had a worse VA at baseline than the eyes of the treatment group. After the antiVEGF therapy an average logMAR BCVA of 0.42 (SD 0.26 , range 0.1-0.8) was assessed in the treatment group, which equates to a decimal VA of 0.4. Every CNV responded well to anti-VEGF treatment and all eyes

Table 2 Morphologic characteristics of 5 traumatic choroidal neovascularizations (CNV) treated with intravitreal anti-VEGF-injections

\begin{tabular}{llllllll}
\hline No. & Location of choroidal rupture & CNV type & CNV location & CNV activity & GLD $(\mu \mathrm{m})$ & Initial CFT $(\mu \mathrm{m})$ & Final CFT $(\mu \mathrm{m})$ \\
\hline 1 & $\sim 1$ PD above optic disc & classic & extrafoveal & active & 451 & 568 & 434 \\
2 & $\sim 1$ PD under optic disc & classic & extrafoveal & active & 1124 & 1240 & 586 \\
3 & $\sim 1$ PD above optic disc & classic & extrafoveal & active & 890 & 329 & 307 \\
4 & $\sim 1$ PD temporal optic disc & classic & subfoveal & active & 1535 & 410 & 214 \\
5 & $\sim 1$ PD temporal optic disc & classic & subfoveal & active & 2725 & 678 & 475 \\
\hline
\end{tabular}

GLD Greatest linear diameter, FFA Fundus fluorescein angiography, CFT Central foveal thickness, VEGF Vascular endothelial growth factor 


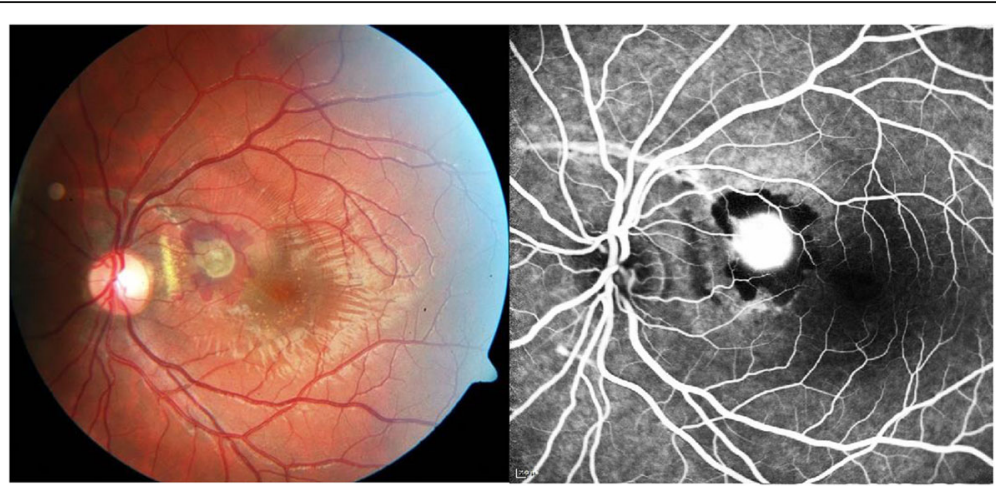

Fig. 1 Traumatic CNV in a 19-year-old male. Colour image and fundus fluorescein angiography (FFA) of a 19-year-old man with traumatic choroidal neovascularization (CNV) in the left eye. Arcuate choroidal rupture can be seen under-crossing the vessels above the optic disc. The greyish CNV with ambient subretinal bleeding, located in the papillomacular region, shows significant leakage on FFA.

presented with improved $(n=4,80 \%)$ or stable VA $(n=$ $1,20 \%)$ after the last anti-VEGF injection. The mean change of BCVA was + 3.8 lines (SD 4.8; range 0-12). Nevertheless, the difference between baseline VA and final VA was statistically not significant $(p=0.152)$. In the group without treatment the mean $\log M A R$ BCVA was 1.1 (SD 0.35, range $0.7-1.4$ ) at the last follow-up appointment. There was no significant change between baseline and follow-up VA in this group $(p=0.939)$. The follow-up period was 62 (SD 32; range 17 - 102) months in the treatment group and 82 months (SD 66; range 6 - 120) in the group without therapy. Table 3 gives a detailed overview of the clinical parameters of the treatment group.

\section{Discussion}

Traumatic choroidal ruptures are seen in 5-10 \% of patients with blunt ocular injuries $[15,16]$. The growth of a secondary trauma-related $\mathrm{CNV}$ is a common complication in such eyes [1]. In our study population $20 \%$ of all patients with traumatic choroidal rupture developed a $\mathrm{CNV}$, which is in agreement with other publications $[3,15]$. In line with other authors [15], we also had a young study population developing traumatic $\mathrm{CNV}$ with an average age of 32 years. Consistent with our data most patients with traumatic chorioretinopathies are male (up to 90\%) [15].

Not every traumatic CNV needs to be treated [15]. The development of fibrovascular tissue and neovascularisation

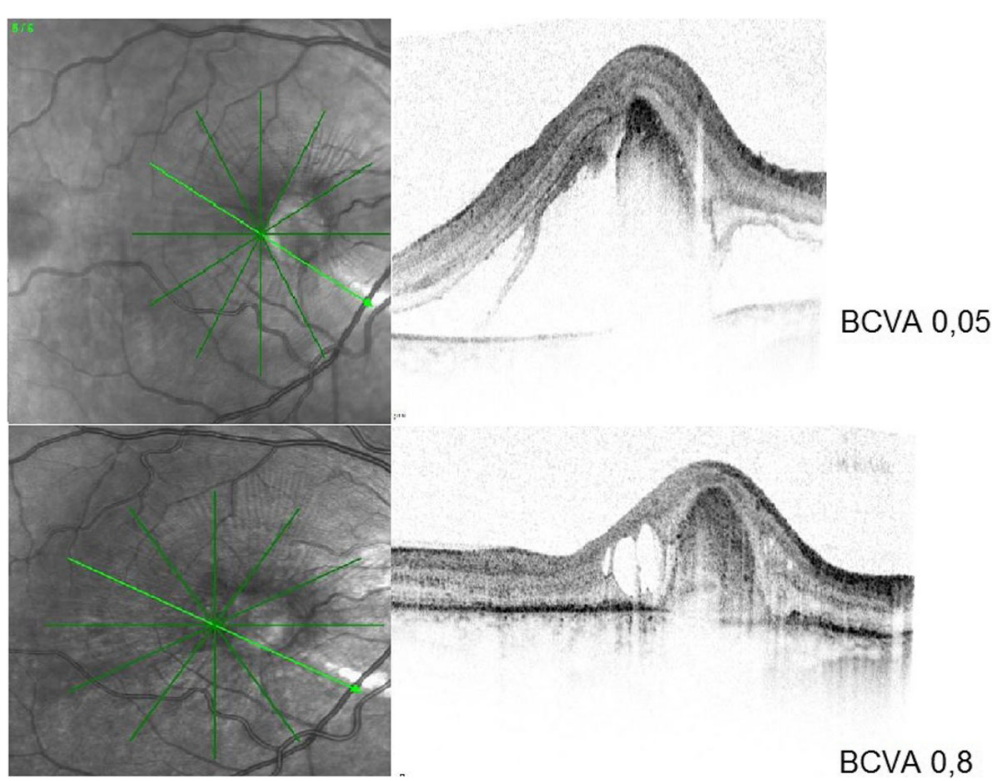

Fig. 2 Regression of traumatic CNV in a 23-year-old male treated with anti-VEGF. Optical coherence tomography images of the right eye of a 19year-old man with traumatic choroidal neovascularization (CNV) a) before treatment and b) four weeks after the last Bevacizumab injection showing regression of CNV. BCVA = best corrected visual acuity on day of retinal imaging 
Table 3 Clinical parameters of 5 eyes with traumatic choroidal neovascularization (CNV) treated with intravitreal anti-VEGF-injections

\begin{tabular}{lllllllll}
\hline No. & age group (yr) & eye & Type of trauma & Anti-VEGF drug & No. of injections & Initial logMAR BCVA & Final logMAR BCVA & $\Delta B C V A$ (lines) \\
\hline 1 & $18-25$ & RE & Penetration & Ranibizumab & 6 & 0.20 & 0.3 & +2 \\
2 & $18-25$ & RE & Contusion & Bevacizumab & 8 & 0.05 & 0.8 & +12 \\
3 & $18-25$ & LE & Contusion & Bevacizumab & 1 & 0.16 & 0.40 & +4 \\
4 & $31-40$ & RE & Contusion & Bevacizumab & 1 & 0.40 & 0.50 & +1 \\
5 & $41-50$ & RE & Contusion & Bevacizumab & 5 & 0.16 & 0.16 & \pm 0 \\
\hline
\end{tabular}

yr Years, RE Right eye, LE Left eye, CNV Choroidal neovascularization, BCVA Best corrected visual acuity, F/U Follow-up, VEGF Vascular endothelial growth factor

is part of the expected healing process and some CNV membranes can undergo watchful observation while regressing $[2,3,15]$. This is consistent with our report of 4 of $11 \mathrm{CNV}$ membranes (36\%) that did not receive further ophthalmic treatment because of inactivity and scarring of the lesion at initial presentation. However, in cases of symptomatic CNV development and activity of the lesion verified by FFA imaging prompt treatment is indicated. Before the anti-VEGF aera secondary CNV membranes were treated with laser photocoagulation [6], photodynamic therapy $[4,5]$ or vitreoretinal surgery [7]. There is nowadays consensus that secondary CNV membranes should receive intravitreal anti-VEGF treatment as first-line-therapy [17].

To our knowledge, only single case reports have been published so far on intravitreal anti-VEGF therapy for traumatic CNV in the past [12-14]. In these cases one injection of either ranibizumab [12] or bevacizumab [13, 14] was administered. However, the follow-up period with a range of 6 to 12 months was quite short compared to our study. This fact could explain the low number of injections given in these individual cases. In our series one case of late recurrence after a six months treatment pause occurred, which emphasizes the need of a thoroughly and adequate long follow-up on these patients. There are also larger retrospective studies with up to 21 cases of intravitreal antiangiogenic treatment of neovascular membranes in younger patients $(<50$ years of age) and from other causes than AMD [8-10]. So far, the MINERVA study is the largest prospective phase III trial to evaluate ranibizumab for treatment of $\mathrm{CNV}$ of uncommon cause. In this study the group of choroidal neovascularization of miscellaneous etiology included 6 patients with choroidal rupture and 1 with posttraumatic etiology, but no separate evaluation of the traumatic CNV group is available [18].

In our patient population with secondary traumatic CNV intravitreal anti-VEGF therapy was effective in the treatment group in respect of functional and anatomical outcome. In every case a good response to anti-VEGF therapy was noted by $\mathrm{CNV}$ regression documented on OCT and FFA imaging. In all treated eyes the CFT was reduced compared to the baseline examination. Every eye showed an improved $(n=4)$ or stable $(n=1)$ BCVA with an average gain of 3.8 BCVA lines. Although the improvement in BCVA $(p=0.152)$ and reduction of CFT ( $p=0.089$ ) was not statistically significant due to the low sample size, we consider the difference of mean values of BVCA and CFT between baseline and final visit as clinically relevant. As expected in the group without anti-VEGF treatment no significant change was noted between baseline and follow-up VA $(p=0.939)$.

Overall, we conclude that in younger individuals with secondary traumatic $\mathrm{CNV}$ the response to intravitreal antiangiogenic treatment seems to be more favourable than in older patients with wet AMD [19]. Congruent with our data, the average count of anti-VEGF injections required to control the activity of the disease is lower compared to patients with AMD-related CNV [18]. Although good results have been achieved with intravitreal antiangiogenic drugs, the lack of long-term data regarding safety and systemic side effects in young individuals has to be emphasized. However, in our study population no serious ocular or systemic side effects were recorded.

\section{Conclusions}

We present the up to now largest retrospective case series with a systematic review of patients with secondary traumatic CNV receiving intravitreal anti-VEGF agents with a mean follow-up of five years. The limitations of our study are its retrospective design, the low sample size, the lack of a standard protocol (different injection regimes and anti-VEGF agents) and the monocentric character. The sample size is compared to other single case reports quite good and every case was well documented including retinal imaging. Further clinical trials with a prospective and randomized character are unlikely to be published with refer to the rarity of this condition.

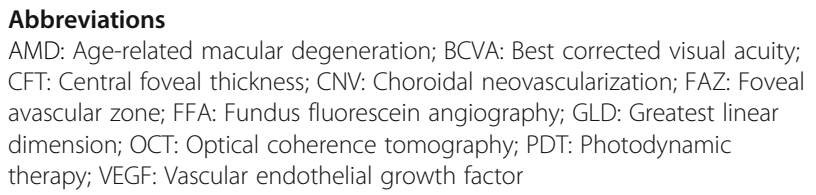

\section{Acknowledgements}

None

Authors' contribution

All authors contributed to the study and the revision of the manuscript. TB: Design of the study, data collection, text writing, design of tables and 
figures; FZ: Statistical expertise. HH, MAG: Critical revision of the manuscript and final approval. All authors have read and approved the manuscript in its current state. All authors declare their consent for publication. All authors read and approved the final manuscript.

\section{Funding}

There was no funding for the study.

\section{Availability of data and materials}

Supporting data can be accessed by contact to the corresponding author (teresa.barth@ukr.de).

The datasets analysed are available from the corresponding author on reasonable request.

\section{Ethics approval and consent to participate}

All procedures performed in the study were in accordance with the ethical standards of the institutional and/or national research committee and with the 1964 Helsinki declaration and its later amendments or comparable ethical standards. Written consent for data processing was obtained from each patient. Our study was approved by the local ethics committee. For further questions please contact ethics committee under the following address: Ethikkommission, Universität Regensburg, 93040 Regensburg, Germany, E-Mail: ethikkommission@ur.de.

\section{Consent for publication}

The clinical datasets were anonymized; individual consent for publication was not required by the ethics committee.

\section{Competing interests}

All authors declare that they have no competing interests.

\section{Author details}

'Department of Ophthalmology, University Medical Centre Regensburg, Franz-Josef-Strauß-Allee, 1193053 Regensburg, Germany. ${ }^{2}$ Centre for clinical studies (ZKS), University Medical Centre Regensburg, Franz-Josef-Strauß-Allee, 1193053 Regensburg, Germany.

Received: 22 March 2019 Accepted: 6 November 2019

Published online: 26 November 2019

\section{References}

1. Wood CM, Richardson J. Chorioretinal neovascular membranes complicating contusional eye injuries with indirect choroidal ruptures. Br J Ophthalmol. 1990;74(2):93-6.

2. Aguilar JP, Green WR. Choroidal rupture: a histopathologic study of 47 eyes. Retina. 1984:4:269-75.

3. Youssri Al, Young LHY. Closed-globe contusion injuries of the posterior segment. Int Ophthalmol Clin. 2002:42(3):79-86.

4. Harissi-Dagher M, Sebag M, Gauthier D, et al. Photodynamic therapy in young patients with choroidal neovascularization following traumatic choroidal rupture. Am J Ophthalmol. 2005;139(4):726-8.

5. Mehta HB, Shanmugam MP. Photodynamic therapy of a posttraumatic choroidal neovascular membrane. Indian J Ophthalmol. 2005;53(2):131-2.

6. Fuller B, Gitter KA. Traumatic choroidal rupture with late serous detachment of macula. Report of successful argon laser treatment. Arch Ophthalmol. 1973;89(4):354-5.

7. Gotzaridis EV, Vakalis AN, Sethi CS, Charteris DG. Surgical removal of sequential epiretinal and subretinal neovascular membranes in a patient with traumatic choroidal rupture. Eye. 2003;17(6):790-1.

8. Carneiro AM, Silva RM, Veludo MJ, et al. Ranibizumab treatment for choroidal neovascularization from causes other than age-related macular degeneration and pathological myopia. Ophthalmologica. 2011;225(2):81-8.

9. Erol MK, Ozdemir O, Coban DT, et al. Ranibizumab treatment for choroidal neovascularization secondary to causes other than age-related macular degeneration with good baseline visual acuity. Semin Ophthalmol. 2014; 29(2):108-13,

10. Gupta B, Elagouz M, Sivaprasad S. Intravitreal bevacizumab for choroidal neovascularisation secondary to causes other than age-related macular degeneration. Eye. 2010;24(2):203-13.

11. Rishi P, Gupta A, Rishi E, Shah BJ. Choroidal neovascularization in 36 eyes of children and adolescents. Eye. 2013;27(10):1158-68
12. Liang F, Puche N, Soubrane G, Souied EH. Intravitreal ranibizumab for choroidal neovascularization related to traumatic Bruch's membrane rupture. Graef Arch Clin Exp. 2009;247(9):1285-8.

13. Rishi $P$, Shroff D, Rishi E. Intravitreal bevacizumab in the management of posttraumatic choroidal neovascular membrane. Retin Cases Brief Rep. 2008; 2(3):236-8.

14. Takahashi M, Kinoshita S, Saito W, et al. Choroidal neovascularization in a patient with blunt trauma-caused traumatic retinopathy without choroidal rupture. Graef Arch Clin Exp. 2011;249(1):137-40.

15. Chen Y, Zhao M, Zhou P. Traumatic chorioretinopathies. In: Ryan SJ, editor. Retina, vol. 2. 5th ed. London, New York, Oxford, St. Louis, Sydney, Toronto: Medical Retina, Saunders; 2012. p. 1564-70.

16. Wyszynski RE, Grossniklaus HE, Frank KE. Indirect choroidal rupture secondary to blunt ocular trauma. A review of eight eyes. Retina. 1988;8(4):237-43.

17. Professional Association of German Ophthalmologists (BVA), German Ophthalmological Society (DOG), German Retina Society e. V. (RG) (2018) Statement of the BVA, the DOG, and the RG on treatment of choroidal neovascularization in diseases other than neovascular age-related macular degeneration: Dated October 2017. Ophthalmologe. 2019;116(Suppl 1):1-9. https:/doi.org/10.1007/s00347-018-0810-1.

18. Lai TYY, Staurenghi G, Lanzetta P, et al. Efficacy and safety of Ranibizumab for the treatment of choroidal neovascularization due to uncommon cause: Twelve-Month Results of the MINERVA Study. Retina. 2018;38(8):1464-77.

19. Spaide RF. Choroidal neovascularization in younger patients. Curr Opin Ophthalmol. 1999;10(3):177-81.

\section{Publisher's Note}

Springer Nature remains neutral with regard to jurisdictional claims in published maps and institutional affiliations.
Ready to submit your research? Choose BMC and benefit from:

- fast, convenient online submission

- thorough peer review by experienced researchers in your field

- rapid publication on acceptance

- support for research data, including large and complex data types

- gold Open Access which fosters wider collaboration and increased citations

- maximum visibility for your research: over $100 \mathrm{M}$ website views per year

At BMC, research is always in progress.

Learn more biomedcentral.com/submissions 